\title{
Phase Transition in Dynamical Systems: Defining Classes of Universality for Two-Dimensional Hamiltonian Mappings via Critical Exponents
}

\author{
Edson D. Leonel \\ Departamento de Estatística, Matemática Aplicada e Computação, \\ Instituto de Geociências e Ciências Exatas, Universidade Estadual Paulista, \\ Av.24A, 1515, Bela Vista, 13506-700, Rio Claro, SP, Brazil \\ Correspondence should be addressed to Edson D. Leonel, edleonel@rc.unesp.br \\ Received 8 April 2009; Accepted 20 July 2009 \\ Recommended by Alexander Loskutov \\ A phase transition from integrability to nonintegrability in two-dimensional Hamiltonian \\ mappings is described and characterized in terms of scaling arguments. The mappings considered \\ produce a mixed structure in the phase space in the sense that, depending on the combination \\ of the control parameters and initial conditions, KAM islands which are surrounded by chaotic \\ seas that are limited by invariant tori are observed. Some dynamical properties for the largest \\ component of the chaotic sea are obtained and described in terms of the control parameters. The \\ average value and the deviation of the average value for chaotic components of a dynamical \\ variable are described in terms of scaling laws, therefore critical exponents characterizing a \\ scaling function that describes a phase transition are obtained and then classes of universality are \\ characterized. The three models considered are: The Fermi-Ulam accelerator model, a periodically \\ corrugate waveguide, and variant of the standard nontwist map.
}

Copyright (C) 2009 Edson D. Leonel. This is an open access article distributed under the Creative Commons Attribution License, which permits unrestricted use, distribution, and reproduction in any medium, provided the original work is properly cited.

\section{Introduction}

Dynamical systems described by two-dimensional nonlinear area preserving mappings have been considered for many years as prototype for the study of low-dimensional physical systems. Applications of the description of dynamical systems by mappings are observed in the study of magnetic field lines in toroidal plasma devices with reversed shear (like tokamaks), channel flows, waveguide, Fermi acceleration and many other [1-10].

In this paper we consider a special class of two-dimensional area preserving mappings exhibiting a phase transition from integrable to nonintegrable. For certain values of the control parameter, the phase space of the system has only periodic and quasiperiodic orbits, thus the phase space is filled by straight lines. As this control parameter varies, the phase 
space exhibits a mixed form containing both Kolmogorov-Arnold-Moser (KAM) islands surrounded by a chaotic sea (conservative chaos) and a set of invariant spanning curves limiting the size of the chaotic sea. This form of the phase space is generic for nondegenerate Hamiltonian systems [11] and can be observed in many different problems including time dependent potential [12-16], tokamaks [17-20] and also in billiards [21-23].

The main approach considered in this paper is the description of the behavior of some average dynamical variables as a function of the control parameter, like the first momenta and the deviation around the average variable. This characterization is made along the chaotic sea near a transition from integrable to nonintegrable. This formalism furnishes critical exponents that can be used to define classes of universality.

This paper is organized as follows. In Section 2, we present and discuss some details needed to construct a generic two-dimensional area preserving mapping. Then application for a specific mapping is made including a discussion of the stability of fixed points and properties of the phase transition from integrable to nonintegrable. In Section 3 the discussion is devoted to a periodically corrugate waveguide. Some results for the phase space are also discussed in connection with the Standard Mapping. The scaling results for the phase transition are obtained and discussed. In Section 4, we extend the applicability of the theory for the one-dimensional Fermi accelerator model confirming that the rather two distinct models discussed in Sections 3 and 4 belong to the same class of universality and experience the same transition. Finally, the conclusion and final remarks are drawn in Section 5.

\section{A Generic Two-Dimensional Area-Preserving Map}

In this section we present and discuss some dynamical properties for a two-dimension mapping. We assume that there is a two-dimensional integrable system that is slightly perturbed. Then, the Hamiltonian function that describes the system is written as

$$
H\left(I_{1}, I_{2}, \theta_{1}, \theta_{2}\right)=H_{0}\left(I_{1}, I_{2}\right)+\varepsilon H_{1}\left(I_{1}, I_{2}, \theta_{1}, \theta_{2}\right),
$$

where the variables $I_{i}$ and $\theta_{i}$ with $i=1,2$ correspond, respectively, to the action and angle. Considering the Poincaré section given by the plane $I_{1} \times \theta_{1}$ assuming that $\theta_{2}$ is constant (mod $2 \pi)$, one can write a modified twist mapping as

$$
T:\left\{\begin{array}{l}
I_{n+1}=I_{n}+\varepsilon h\left(\theta_{n}, I_{n+1}\right) \\
\theta_{n+1}=\left[\theta_{n}+K\left(I_{n+1}\right)+\varepsilon p\left(\theta_{n}, I_{n+1}\right)\right] \quad \bmod (2 \pi)
\end{array}\right.
$$

where both $h, K$, and $p$ are, in principle, nonlinear functions of their variables and the index $n$ correspond to the $n$th iteration of the mapping. The variables $\theta$ and $I$ corresponds indeed to $I_{1}$ and $\theta_{1}$.

Since the mapping (2.2) must be area preserving (it was obtained from an Hamiltonian) then the functions $h\left(\theta_{n}, I_{n+1}\right)$ and $p\left(\theta_{n}, I_{n+1}\right)$ must obey some intrinsic relations. 
The relations are obtained considering that the determinant of the Jacobian matrix is the unity. Then we have that the coefficients are written as

$$
\begin{aligned}
\frac{\partial I_{n+1}}{\partial I_{n}} & =\frac{1}{1-\varepsilon\left(\partial h\left(\theta_{n}, I_{n+1}\right) / \partial I_{n+1}\right)}, \\
\frac{\partial I_{n+1}}{\partial \theta_{n}} & =\varepsilon \frac{\partial h\left(\theta_{n}, I_{n+1}\right)}{\partial \theta_{n}}+\varepsilon \frac{\partial h\left(\theta_{n}, I_{n+1}\right)}{\partial I_{n+1}} \frac{\partial I_{n+1}}{\partial \theta_{n}}, \\
\frac{\partial \theta_{n+1}}{\partial I_{n}} & =\left[\frac{\partial K\left(I_{n+1}\right)}{\partial I_{n+1}}+\varepsilon \frac{\partial p\left(\theta_{n}, I_{n+1}\right)}{\partial I_{n+1}}\right] \frac{\partial I_{n+1}}{\partial I_{n}}, \\
\frac{\partial \theta_{n+1}}{\partial \theta_{n}} & =1+\varepsilon \frac{\partial p\left(\theta_{n}, I_{n+1}\right)}{\partial \theta_{n}}+\left[\frac{\partial K\left(I_{n+1}\right)}{\partial I_{n+1}}+\varepsilon \frac{\partial p\left(\theta_{n}, I_{n+1}\right)}{\partial I_{n+1}}\right] \frac{\partial I_{n+1}}{\partial \theta_{n}} .
\end{aligned}
$$

Given the above coefficients, the determinant of the Jacobian matrix is given by

$$
\text { Det } J=\frac{\left[1+\varepsilon\left(\partial p\left(\theta_{n}, I_{n+1}\right) / \partial \theta_{n}\right)\right]}{\left[1-\varepsilon\left(\partial h\left(\theta_{n}, I_{n+1}\right) / \partial I_{n+1}\right)\right]}
$$

It is easy then to conclude that area preservation will be observed only if the condition

$$
\frac{\partial p\left(\theta_{n}, I_{n+1}\right)}{\partial \theta_{n}}+\frac{\partial h\left(\theta_{n}, I_{n+1}\right)}{\partial I_{n+1}}=0
$$

is matched. For many mappings, the function $p\left(\theta_{n}, I_{n+1}\right)=0$. Therefore, if we keep the function $h$ as $h\left(\theta_{n}\right)=\sin \left(\theta_{n}\right)$, just to illustrate applicability of the formalism, the following mappings have already been studied:

(i) $K\left(I_{n+1}\right)=I_{n+1}$, then the Taylor-Chirikov's map is recovered;

(ii) $K\left(I_{n+1}\right)=2 / I_{n+1}$, then the Fermi-Ulam accelerator model is obtained;

(iii) $K\left(I_{n+1}\right)=\zeta I_{n+1}$, with $\zeta$ constant, then the bouncer model is found;

(iv) for the case of

$$
K\left(I_{n+1}\right)= \begin{cases}4 \zeta^{2}\left(I_{n+1}-\sqrt{I_{n+1}^{2}-\frac{1}{\zeta^{2}}}\right), & \text { if } I_{n+1}>\frac{1}{\zeta^{\prime}} \\ 4 \zeta^{2} I_{n+1}, & \text { if } I_{n+1} \leq \frac{1}{\zeta}\end{cases}
$$

where $\zeta$ is a constant, then we recovered the so-called Hybrid-Fermi-Ulam-bouncer model;

(v) considering $K\left(I_{n+1}\right)=I_{n+1}+\zeta I_{n+1}^{2}$, the logistic twist mapping is obtained. 


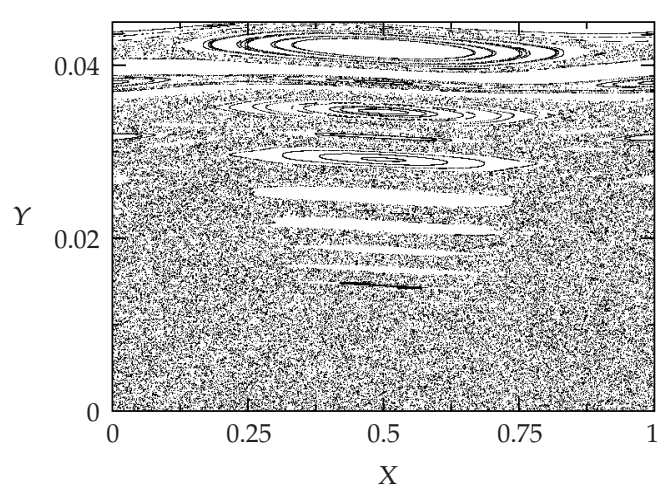

(a)

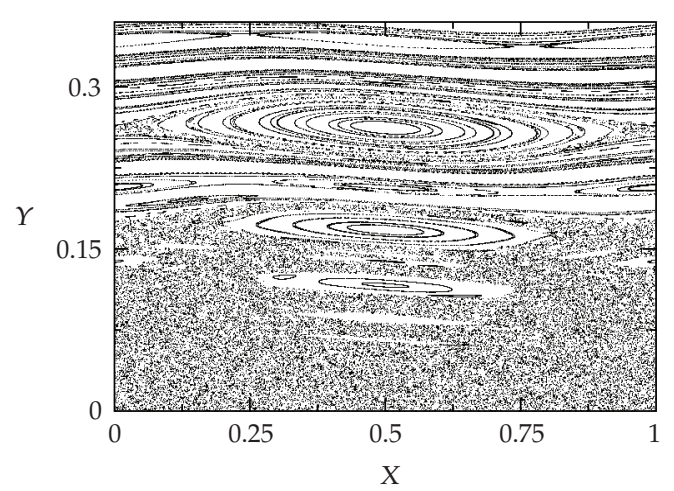

(b)

Figure 1: Phase space generated by mapping (2.7) for the control parameters $a=2$ and: (a) $b=10^{-3}$ and (b) $b=10^{-2}$.

In this section, we shall consider the following expression for the two-dimensional area-preserving mapping:

$$
T:\left\{\begin{array}{l}
y_{n+1}=\left|y_{n}-b \sin \left(2 \pi x_{n}\right)\right|, \\
x_{n+1}=\left[x_{n}+\frac{a}{\sqrt{y_{n+1}}}\right] \bmod (1),
\end{array}\right.
$$

where the variables $I$ and $\theta$ were replaced, by sake of simplicity, by $y$ and $x$. The control parameters of the model are $a$ and $b$. The modulus function is requested since the argument of the denominator of the second equation above is not defined for nonpositive values. It is easy to see that when the control parameter $b=0$, then the system is integrable. For $b \neq 0$, the phase space of the system exhibits chaotic components and periodic orbits, as we can see in Figure 1. The determinant of the Jacobian matrix of the mapping (2.7) is the unity, so area preservation is observed.

The fixed points of the mapping (2.7) are obtained by matching the following conditions: $y_{n+1}=y_{n}=y$ and $x_{n+1}=x_{n}=x+m$ with $m=1,2,3, \ldots$ After solving these two conditions, the two sets of fixed points are given by: fixed point $1(x, y)=\left(0, a^{2} / m^{2}\right)$; fixed point $2(x, y)=\left(1 / 2, a^{2} / m^{2}\right)$. The fixed point 1 is always hyperbolic while fixed point 2 is elliptic for

$$
a>\sqrt[4]{\frac{b^{2} \pi^{2} m^{6}}{16}}
$$

From now on, we shall consider as fixed the control parameter $a=2$. Results for $a \neq 0$ will be presented elsewhere.

We can see in Figure 1 that the size of the chaotic sea varies as the control parameter $b$ varies. As $b$ rises, the position of the lowest invariant spanning curve also rises. It is then expected that the average value of $y$, say $\bar{y}$ changes as a function of the control parameter. To 




(a)

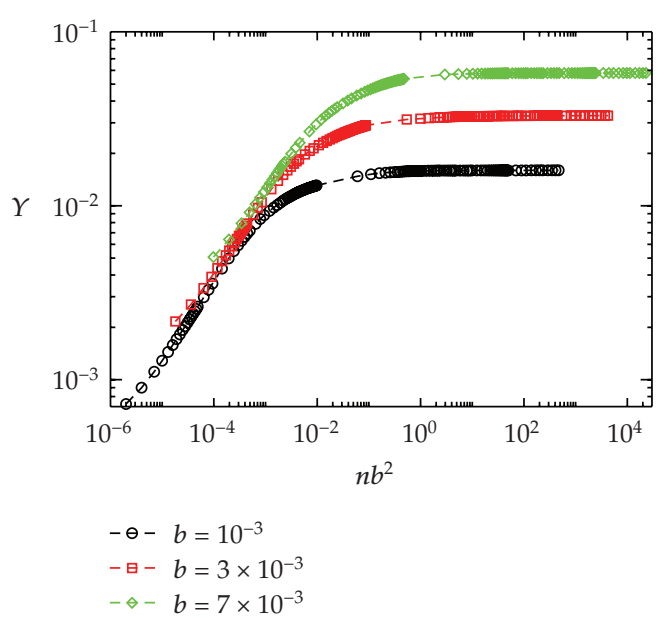

(b)

Figure 2: Behavior of $\bar{y}$ as function of $n$ for the following control parameters: (a) $b=10^{-3}, b=3 \times 10^{-3}$ and $b=7 \times 10^{-3}$; (b) same plot of (a) after a transformation $n \rightarrow n b^{2}$.

obtain the average value of $y$, we proceed as follows. First we obtain the average value along an orbit

$$
y(n, b)=\frac{1}{n} \sum_{i=1}^{n} y_{i}
$$

and then we evolve the average over an ensemble of $M$ different initial conditions along the chaotic sea. The average value is then written as

$$
\bar{y}(n, b)=\frac{1}{M} \sum_{j=1}^{M} y_{j}
$$

where $y_{j}$ is a sample of the ensemble. It is shown in Figure 2 the behavior of $\bar{y} \times n$ for three different control parameters. We can see that $\bar{y}$ starts growing and then, after reaching a characteristic crossover iteration number, it bends towards a regime of convergence. We see also that the three curves start growing at different positions. Therefore, a transformation $n \rightarrow n b^{2}$ coalesces all curves at small iterations, as we can see in Figure 2(b).

The behavior shown in Figure 2 allow us to suppose the following.

(i) For low iterations and after a short initial transient, that is, $1 \ll n \ll n_{x}$, the average $y$ is described as $\bar{y} \alpha\left(n b^{2}\right)^{\beta}$, where $\beta$ is a critical exponent.

(ii) For large enough $n$, say $n \gg n_{x}$, then $\bar{y}_{\text {sat }} \alpha b^{\alpha}$, where $\alpha$ is a critical exponent.

(iii) The number of iterations which characterizes the changeover from growth to the saturation is given $n_{x} \propto b^{z}$ where $z$ is also a critical exponent.

Plotting the behavior of $\bar{y}_{\text {sat }} \times b$ and $n_{x} \times b$ we can obtain the critical exponents $\alpha$ and $z$. The corresponding plots of $\bar{y}_{\text {sat }} \times b$ and $n_{x} \times b$ are shown in Figure 3. After applying a power law 


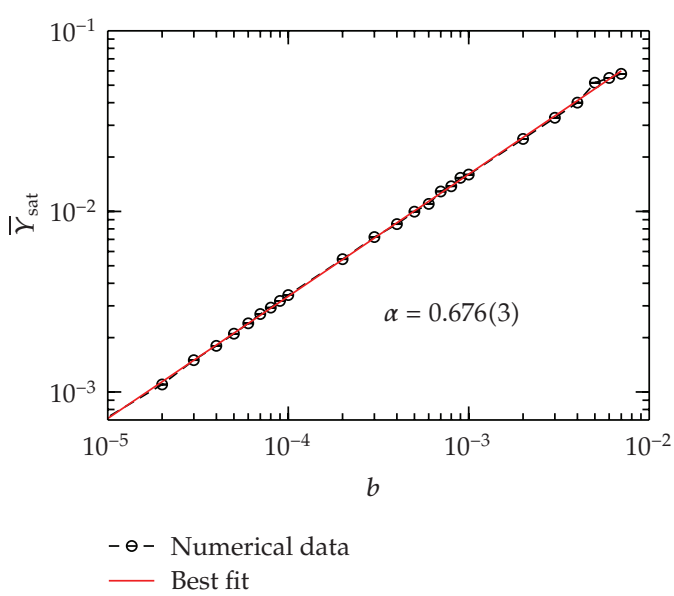

(a)

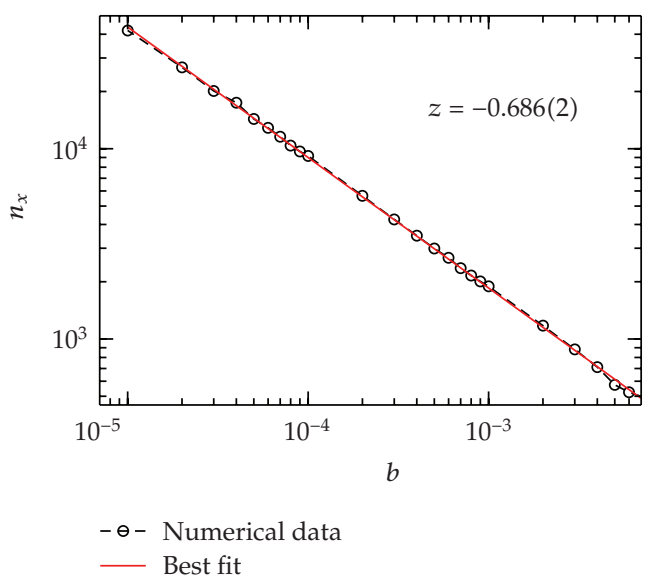

(b)

Figure 3: Plot of: (a) $\bar{y}_{\text {sat }} \times b$ and (b) $n_{x} \times b$. A power law fitting furnishes that $\alpha=0.676(3)$ and $z=-0.686(2)$.

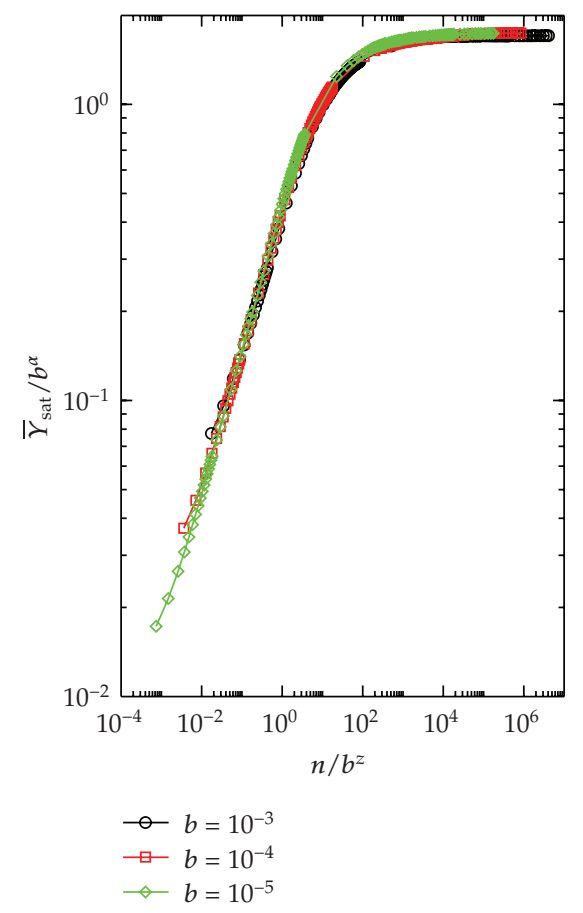

Figure 4: Collapse of different curves of $\bar{y}$, after a suitable rescale in the axis, into a single and universal plot.

fitting we obtain that $\alpha=0.676(3)$ and $z=-0.686(2)$. The critical exponent $\beta$ was obtained for several different time series. Its average value is around $\beta \approx 0.5$.

Rescaling properly, we can see that all curves coalesce together, as they are shown in Figure 4. Such collapse is a clear evidence of the criticality for the chaotic sea near a transition from integrability to nonintegrability. It must be emphasized that the values obtained for 
the critical exponents are useful to define classes of universality. As we will see in the two next sections, for the periodically corrugate waveguide and for the one-dimensional Fermi accelerator model, the exponents obtained here are different from the critical exponents obtained there, thus confirming that, despite the similarity of the two-dimensional mappings, the classes of universality of such models are different.

\section{Periodically Corrugate Waveguide}

Let us now discuss in this section some properties of a periodically corrugated waveguide. This section is a review of papers $[24,25]$. The interest in describing the problem of guiding a light ray inside a periodically corrugated boundary has increased along last years, mainly because the topic is applicable in so many different fields of science. Applications involve, for example, ray chaos in underwater acoustics [26-28], quantum transport in ballistic cavities $[29,30]$, transport through a finite $\mathrm{GaAs} / \mathrm{Al}_{\mathrm{x}} \mathrm{Ga}_{1-\mathrm{x}} \mathrm{As}$ hetero-structure [31], quantized ballistic conductance in a periodically modulated quantum channel [32], comparisons of classical versus quantum behavior in periodic mesoscopic systems [33, 34], scattering of a quantum particle in a rippled waveguide [35] and anomalous wave transmittance in the stop band of a corrugated parallel-plane waveguide [36].

There are many different ways to describe problems involving waveguides. One of them consists in considering the well-known billiard approach (A billiard problem consists of a system in which a point-like particle moves freely inside a bounded region and suffers specular reflections with the boundaries.). Generally, the dynamics is described using the formalism of discrete maps. Therefore, depending on the combinations of the control parameters as well as on the initial conditions, the phase space for such mappings might be included in three different classes namely: (i) regular, (ii) ergodic, and (iii) mixed. Roughly speaking, the integrability of the regular cases is generally related to the angular momentum preservation and the static circular billiard is a typical example. On the other hand, for the completely ergodic billiards, only chaotic and therefore unstable periodic orbits are present in the dynamics. The well-known Bunimovich stadium [37] and the Sinai billiard [38] (sometimes also-called as Lorenz gas) are typical examples of case (ii). On these two kinds of systems, the time evolution of a single initial condition, for the appropriated combinations of control parameters, is enough to fill up ergodically the entire phase space. Finally, there are many different billiards that present mixed phase space structure [39-43], which have control parameters with different physical significance. Depending on the combination of both initial conditions and control parameters, the phase space presents a very rich structure which contains invariant spanning curves (sometimes also-called as invariant tori), KAM islands and chaotic seas.

In this section, scaling arguments are used to describe the behavior of the deviation of the average reflection angle within the chaotic sea, for the problem of a classical light ray inside a periodically corrugated waveguide suffering specular reflections. The model consists of a classical light ray which is specularly reflected between a flat plane at $y=0$ and a corrugated surface given by $y=y_{0}+d \cos (k x)$, where $y_{0}$ is the average distance between the flat plane and the corrugated surface, $d$ is the amplitude of the corrugation and $k$ is the wave number. The dynamical variables, which are used in the description of the problem are the angle $\theta$ of the trajectory measured from the positive horizontal axis and the corresponding value of the coordinate $x$ at the instant of the reflection. It must be emphasized that the mapping is iterated when the ray light hits the flat plane, thus multiple reflections with 


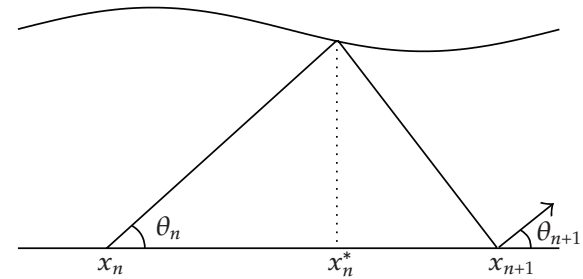

(a)

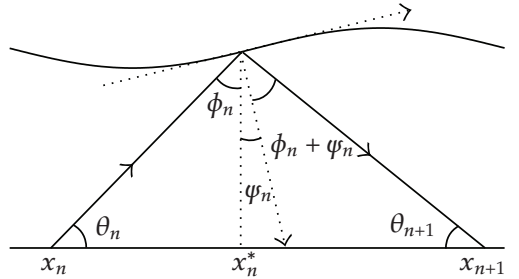

(b)

Figure 5: (a) Reflection from the corrugated surface of a light ray coming from the flat surface at $y=0$. (b) Details of the trajectory before and after a collision with the corrugated surface.

the periodically corrugated surface are neglected. (This is a standard approximation which is very useful to speed up the numerical simulations since no transcendental equations must be solve, as they have to be in the corresponding complete version of the problem).

\subsection{The Model and the Mapping}

Let us discuss now all the details needed to describe the model and obtain the equations that describe the dynamics of the problem. The model thus consists in obtaining a mapping $T\left(\theta_{n}, x_{n}\right)=\left(\theta_{n+1}, x_{n+1}\right)$, given the initial conditions $\left(\theta_{n}, x_{n}\right)$ as shown in Figure 5(a). The mapping is obtained from purely geometrical considerations of Figure $5(b)$. It is easy to see that

$$
x_{n}^{*}-x_{n}=\frac{\left(y_{0}+d \cos \left(k x_{n}^{*}\right)\right)}{\theta_{n}} .
$$

and in a similar way

$$
x_{n+1}-x_{n}^{*}=\frac{\left(y_{0}+d \cos \left(k x_{n}^{*}\right)\right)}{\theta_{n+1}} .
$$

We stress that the term $x_{n}^{*}$ gives the exact location of the collision on the corrugated surface. On the other hand, the angle $\theta_{n}$ is written as

$$
\theta_{n+1}=\theta_{n}-2 \psi_{n}
$$

where $\tan \left(\psi_{n}(x)\right)=d y(x) / d x=-d k \sin \left(k x_{n}^{*}\right)$ gives the slope of the corrugated surface at the point $x=x_{n}^{*}$. The condition of specular reflection (A specular reflection is that in which the angle of incidence is equals to the angle of reflection. See details in Figure 5(b)) was used in the derivation of the (3.3). The Equations (3.2) and (3.3) correspond to the exact form of the mapping. However, I will discuss in this paper only a simplified version. To do this, the following approximations are assumed.

(1) The relative corrugation is assumed to be small, so that $d / y_{0} \ll 1$, yielding consequently $y_{0}+d \cos \left(k x_{n}^{*}\right) \cong y_{0}$.

(2) In the limit of small corrugation, it is also assumed that $\tan \left(\psi_{n}\right) \cong \psi_{n}$. 


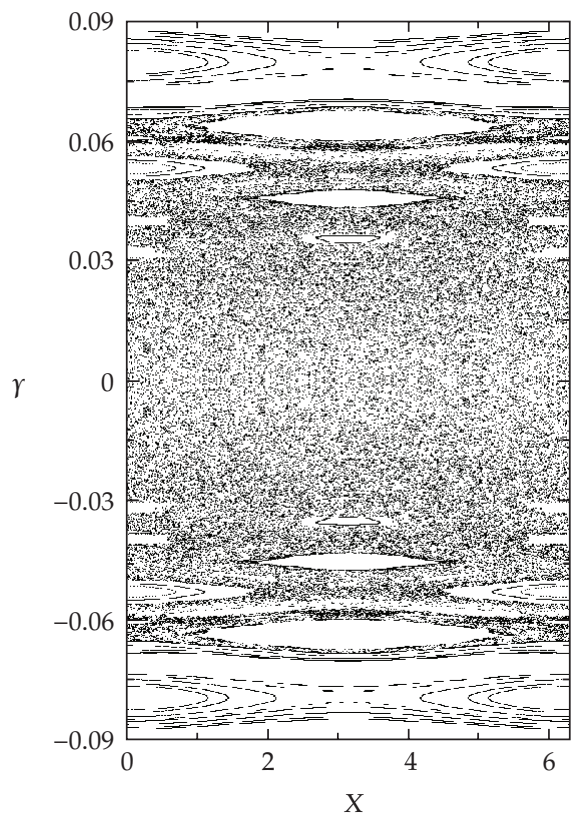

Figure 6: Phase space for the mapping (3.5) with the control parameter $\delta=10^{-3}$.

Let us now argue on the approximation of small relative corrugation. It is easy to see that, for $d=0$, the system is integrable, since the two boundaries are parallel plates and the phase space shows only straight lines. However, if $d \neq 0$ the phase space birth as mixed therefore containing both chaos, invariant spanning curves and KAM islands. Thus, there is an abrupt transition from integrability to nonintegrability when the control parameter goes from $d=0$ to $d \neq 0$. This is the transition we shall address in this section. We stress also that the formalism can be extendable to many other systems and even to other different kinds of transitions. Before write the equations of the mapping, we can see that there are an excessive number of control parameters, namely $d, k, y_{0}$. The dynamics however does not depend on all of them. It is therefore convenient to define the following dimensionless and more convenient variables $\delta=d / y_{0}, \gamma_{n}=\theta_{n} / k$ and finally $X_{n}=k x_{n} / y_{0}$. Thus the only relevant control parameter is $\delta$. With these new variables, the two-dimensional nonlinear mapping is given by

$$
T:\left\{\begin{array}{l}
X_{n+1}=X_{n}+\left[\frac{1}{\gamma_{n}}+\frac{1}{\gamma_{n+1}}\right] \bmod (2 \pi), \\
\gamma_{n+1}=\gamma_{n}+2 \delta \sin \left(X_{n}+\frac{1}{\gamma_{n}}\right) .
\end{array}\right.
$$

This mapping preserves the phase space measure since that the determinant of the Jacobian matrix is the unity.

It is shown in Figure 6, the phase space generated by the iteration of mapping (3.4). We can see that there is a complex structure which contains KAM islands surrounded by a large chaotic sea that is confined between two invariant spanning curves. The chaotic sea was characterized using the well-known Lyapunov exponent. Such exponent shows great 
applicability as a practical tool that can quantify the average expansion or contraction rate for a small volume of initial conditions. As discussed in [44], the Lyapunov exponents are defined as

$$
\lambda_{j}=\lim _{n \rightarrow \infty} \frac{1}{n} \ln \left|\Lambda_{j}\right|, \quad j=1,2
$$

where $\Lambda_{j}$ are the eigenvalues of $M=\prod_{i=1}^{n} J_{i}\left(\gamma_{i}, X_{i}\right)$, and $J_{i}$ is the Jacobian matrix evaluated over the orbit $\left(\gamma_{i}, X_{i}\right)$. It is important to stress that a direct implementation of a computational algorithm to evaluate (3.5) has a severe limitation to obtain $M$. Even in the limit of very short $n$, the components of the matrix $M$ can assume very different orders of magnitude for chaotic orbits and periodic attractors, yielding impracticably the implementation of the algorithm. In order to avoid such problem, we note that $J$ can be written as $J=\Theta T$ where $\Theta$ is an orthogonal matrix and $T$ is a right triangular matrix. Thus we rewrite $M$ as $M=$ $J_{n} J_{n-1} \ldots J_{2} \Theta_{1} \Theta_{1}^{-1} J_{1}$, where $T_{1}=\Theta_{1}^{-1} J_{1}$. A product of $J_{2} \Theta_{1}$ defines a new $J_{2}^{\prime}$. In a next step, it is easy to show that $M=J_{n} J_{n-1} \cdots J_{3} \Theta_{2} \Theta_{2}^{-1} J_{2}^{\prime} T_{1}$. The same procedure can be used to obtain $T_{2}=\Theta_{2}^{-1} J_{2}^{\prime}$ and so on. Using this procedure, the problem is reduced to evaluate the diagonal elements of $T_{i}: T_{11}^{i}, T_{22}^{i}$. Finally, the Lyapunov exponents are now given by

$$
\lambda_{j}=\lim _{n \rightarrow \infty} \frac{1}{n} \sum_{i=1}^{n} \ln \left|T_{j j}^{i}\right|, \quad j=1,2
$$

If at least one of the $\lambda_{j}$ is positive then the orbit is classified as chaotic. Therefore we define $\lambda$ as the larger value of the $\lambda_{j}$. It is shown in Figure 7 the behavior of the positive Lyapunov exponent for an ensemble of 10 different initial conditions on the chaotic sea for the control parameter $\delta=10^{-3}$. The initial conditions were chosen such that $X_{0}=\delta$ and 10 different initial values of $\gamma_{0}$ were uniformly distributed along the interval $\gamma_{0} \in[0,2 \pi]$. Each initial condition was iterated up to $5 \times 10^{8}$ times. The average value obtained was $\bar{\lambda}=1.624(7)$ where the error 0.007 corresponds to the standard deviation of the 10 samples.

Let us now discuss one of the consequences of the variation of the control parameter $\delta$ on the location of the invariant spanning curves. Since the two first invariant spanning curves define the limit for the chaotic sea, the variation of $\delta$ consequently change the edges of the chaotic sea. Before do that, I must first give a brief review in the well-known Standard Map (see also [45] for specific details). It is defined as

$$
T:\left\{\begin{array}{l}
I_{n+1}=I_{n}+K \sin \left(\Theta_{n}\right), \\
\Theta_{n+1}=\Theta_{n}+I_{n+1},
\end{array}\right.
$$

where $K$ is a control parameter. This mapping can also shows mixed phase space for $K \neq 0$. Moreover, one of the most important properties is that it shows a transition from local to global chaos (see $[45,46]$ for specific details) when the control parameter matches the condition $K>0.971 \ldots$... After this transition, no invariant spanning curves are present and consequently the chaotic sea spreads over the phase space. The connection of this result with the phase space of the mapping that describes the behavior of the light ray in a periodically corrugate waveguide suppose that, near the invariant spanning curves that limit the chaotic 


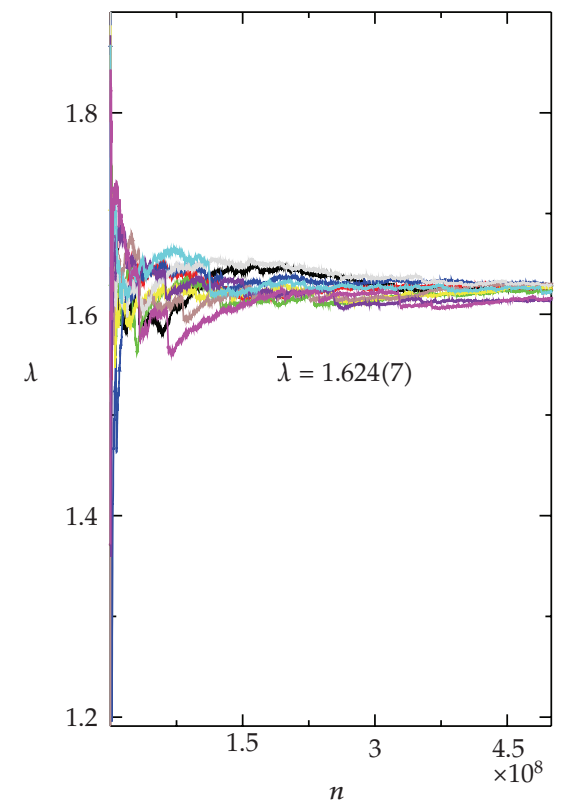

Figure 7: The positive Lyapunov exponent obtained via triangularization algorithm for the control parameter $\delta=10^{-3}$.

sea, the dynamics could be locally described by using the standard mapping. Thus we can suppose that near the invariant spanning curve, the reflection angle can be written as

$$
\gamma_{n+1} \cong \gamma^{*}+\Delta \gamma_{n+1}
$$

where $\gamma^{*}$ is a typical value of the reflection angle along the invariant spanning curve, and $\Delta \gamma_{n+1}$ is a small perturbation of the angle. After defining $Z_{n}=X_{n}+1 / \gamma_{n}$, the first equation of the mapping (3.4) is written as

$$
Z_{n+1}=Z_{n}+\frac{2}{\gamma_{n+1}}
$$

Using (3.8), we can rewrite (3.9) as

$$
Z_{n+1}=Z_{n}+\frac{2}{r^{*}}\left[1+\frac{\Delta \gamma_{n+1}}{r^{*}}\right]^{-1}
$$

Expanding (3.10) in Taylor series, we obtain that

$$
Z_{n+1}=Z_{n}+\frac{2}{r^{*}}\left[1-\frac{\Delta \gamma_{n+1}}{r^{*}}+O\left(\frac{\Delta \gamma_{n+1}}{r^{*}}\right)^{2}\right]
$$


Considering the first representative term in the series, we obtain that

$$
Z_{n+1}=Z_{n}+\frac{2}{r^{*}}\left[1-\frac{\Delta \gamma_{n+1}}{r^{*}}\right]
$$

On the other hand, the second equation of mapping (3.4) may be rewritten as

$$
\gamma^{*}+\Delta \gamma_{n+1}=\gamma^{*}+\Delta \gamma_{n}+2 \delta \sin \left(Z_{n}\right)
$$

Multiplying both sides of (3.13) by $-2 / \gamma^{* 2}$ and adding again in both sides the term $2 / \gamma^{*}$, defining the term

$$
I_{n+1}=-\frac{2 \Delta \gamma_{n+1}}{r^{* 2}}+\frac{2}{r^{*}}
$$

and calling $\phi_{n}=Z_{n}+\pi$, we obtain the corresponding mapping

$$
T:\left\{\begin{array}{l}
I_{n+1}=I_{n}+\frac{4 \delta}{r^{* 2}} \sin \left(\phi_{n}\right), \\
\phi_{n+1}=\phi_{n}+I_{n+1} .
\end{array}\right.
$$

An immediate comparison of (3.7) and (3.15) allow us to conclude that there is an effective control parameter given by

$$
K_{\mathrm{eff}}=\frac{4 \delta}{r^{* 2}}
$$

Since the transition from local to global chaos occurs at $K_{\text {eff }} \cong 0.971 \ldots$, we obtain that the two invariant spanning curves that limit the chaotic sea are given by

$$
r^{*} \cong \pm 2 \sqrt{\frac{\delta}{0.971 \ldots}} .
$$

Therefore we can conclude that the size of the chaotic sea is proportional to $\sqrt{\delta}$ [25].

\subsection{Scaling Approach}

This section is devoted to discuss a scaling property which is present in the chaotic sea. As it was discussed in Section 2, an initial condition in the region of the chaotic sea wanders in a chaotic way along the accessible region, however it is always confined between two invariant spanning curves. The location of the first positive and negative invariant spanning curves depend on the value of the control parameter $\delta$. As a consequence, the "amplitude" 


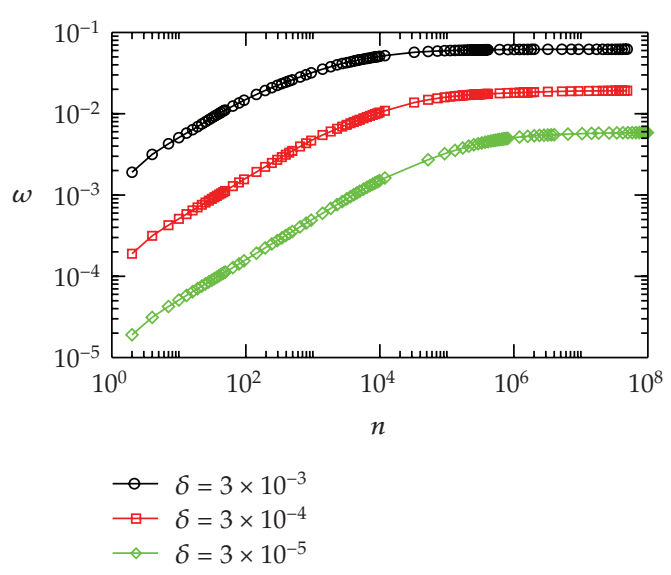

(a)

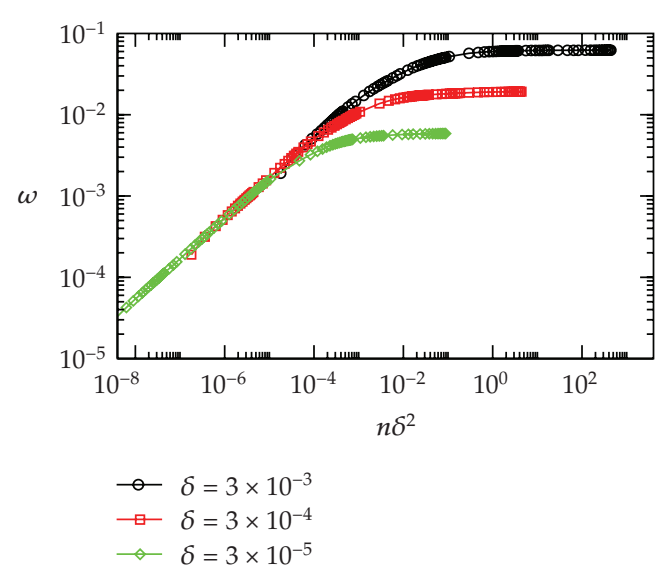

(b)

Figure 8: (a) Plot of $\omega$ as function of the iteration number $n$ for three different control parameters. (b) After a suitable changing of variables, all three curves start growing together.

of a chaotic time series is dependent on the control parameter too. We will now explore the behavior of the deviation of the average value for the angle $r$, an observable defined as

$$
\omega(n, \delta)=\frac{1}{M} \sum_{i=1}^{M} \sqrt{\bar{r}_{i}^{2}(n, \delta)-\bar{\gamma}_{i}^{2}(n, \delta)}
$$

where it is used as

$$
\bar{\gamma}(n, \delta)=\frac{1}{n} \sum_{i=0}^{n} \gamma_{i}
$$

The (3.19) gives the correspondent average reflection angle averaged over the orbit, while the $M$ in (3.18) corresponds to an ensemble of different initial conditions. Therefore, (3.18) was iterated using an ensemble of $M=5 \times 10^{3}$ different initial conditions. The variable $\gamma_{0}$ was kept fixed as $\gamma_{0}=10^{-2} \delta$ while the 5000 values of $X_{0}$ were uniformly distributed along $X_{0} \in[0,2 \pi)$. It is shown in Figure 8(a) the behavior of three different values of $\omega$ for different control parameters. It is easy to see that $\omega$ grows for short iterations and then suddenly it bends towards a regime of saturation for large enough iterations. The changeover from growth to the saturation regime is marked by a characteristic crossover iteration number denoted as $n_{x}$. It is worth stressing that different values for the control parameters generate different curves for $\omega$ for short iteration $n$. This might be an indicative that $n$ is not a "good" scaling variable. Thus, a trick transformation $n \rightarrow n \delta^{2}$ coalesces all the curves for short iteration number, as can be seen in Figure 8(b). Based on the behavior of Figure 8, the following three scaling hypotheses are proposed. 
(i) For a short iteration number, say $n \ll n_{x}$, $\omega$ behaves according to the power law

$$
\omega\left(n \delta^{2}, \delta\right) \propto\left(n \delta^{2}\right)^{\beta}
$$

where $\beta$ is a critical exponent.

(ii) For large enough iteration number, say $n \gg n_{x}, \omega$ approaches a regime of saturation marked by a constant "plateau" given by

$$
\omega_{\text {sat }} \propto \delta^{\alpha},
$$

where the exponent $\alpha$ is a critical exponent;

(iii) The number of iterations that characterizes the crossover, that is, the iterations that marks the change from growth to the saturation is written as

$$
n_{x} \propto \delta^{z}
$$

The exponent $z$ is defined as the dynamical exponent.

This scaling formalism is commonly used in the description of phase transitions in critical phenomena. It is also very useful in studies of problems of surface sciences (see, e.g., [47]). Considering these three initial suppositions, $\omega$ can now be formally described in terms of a scaling function of the type

$$
\omega\left(n \delta^{2}, \delta\right)=l \omega\left(l^{a} n \delta^{2}, l^{b} \delta\right)
$$

where $l$ is a scaling factor, $a$ and $b$ are the so-called scaling exponents. Moreover, the exponents $a$ and $b$ must be necessarily related to the critical exponents $\alpha, \beta$ and $z$. Since $l$ is a scaling factor, it could be chosen that $l=\left(n \delta^{2}\right)^{(-1 / a)}$ and then (3.23) is rewritten as

$$
\omega\left(n \delta^{2}\right)=\left(n \delta^{2}\right)^{(-1 / a)} \omega_{1}\left(\left[n \delta^{2}\right]^{(-b / a)} \delta\right)
$$

where the function $\omega_{1}=\omega\left(1,\left[n \delta^{2}\right]^{-b / a} \delta\right)$ is assumed to be constant on the limit of $n \ll n_{x}$. A comparison of (3.24) and (3.20) allows us to conclude that $\beta=-1 / a$. Choosing now that $l=\delta^{-1 / b},(3.23)$ is given by

$$
\omega\left(n \delta^{2}, \delta\right)=\delta^{-1 / b} \omega_{2}\left(\delta^{-(a / b)} n \delta^{2}\right)
$$

where the function $\omega_{2}=\omega\left(\delta^{-(a / b)} n \delta^{2}, 1\right)$ is assumed to be constant for $n \gg n_{x}$. Comparing now (3.25) and (3.21), it is easy to see that $\alpha=-1 / b$. Given the two different expressions of the scaling factor $l$, we can obtain the relation between the critical exponents as

$$
z=\frac{\alpha}{\beta}-2
$$




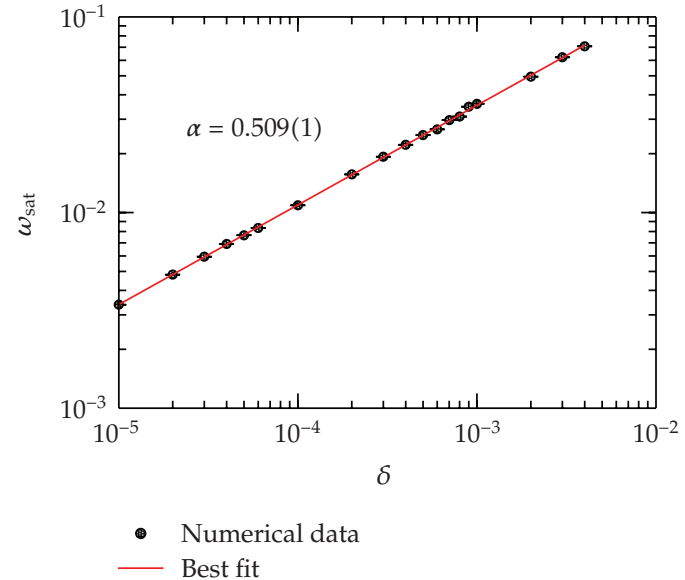

(a)

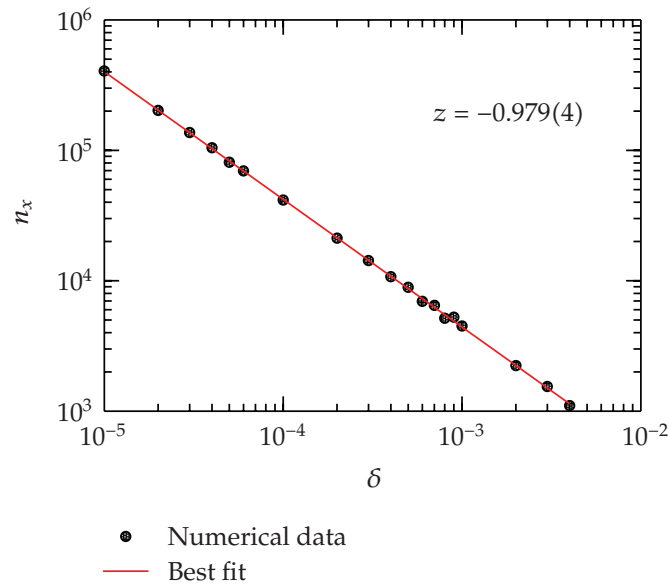

(b)

Figure 9: Plot of (a) $\omega_{\text {sat }}$ and (b) crossover iteration number $n_{x}$ as function of the control parameter $\delta$.

Note however that the scaling exponents are all determined if the critical exponents $\alpha$ and $\beta$ were numerically obtained. Thus, it is shown in Figure 9 the behavior of (a) $\omega_{\text {sat }} \times \delta$ and (b) $n_{x} \times \delta$. The point which is important to stress is that the saturation values were obtained via extrapolation since that even after almost $10^{3} n_{x}$, the saturation value for $\omega$ has not yet been reached. A power law on Figures 9(a) and 9(b) gives that $\alpha=0.509(1), \beta=0.4997(8)$ and $z=-0.979(4)$. Since the dynamical exponent $z$ can also be obtained of (3.26), from evaluation of the numerical values of $\alpha$ and $\beta$, it was found that $z=-0.9814$ (3). Such result is indeed in good agreement with the numerical result obtained in Figure 9(b).

Given that the values of the critical exponents are now obtained, the scaling hypotheses can be verified. In this sense, it is shown in Figure 10 the collapse for three different $\omega$ curves generated from different values of the control parameters onto a single and universal plot. The control parameters used in Figure 10(a) were $\delta=3 \times 10^{-3}, \delta=3 \times 10^{-4}$ and $\delta=3 \times 10^{-5}$. The collapse obtained in Figure 10(b) reinforces that the scaling suppositions are indeed correct and therefore confirms a criticality near $\delta=0$.

\section{One-Dimensional Fermi Accelerator Model}

In this section we will use the formalism described in Section 3 to characterize the same transition in a rather different model, the well-known one-dimensional Fermi accelerator model (FAM). This section is a short review of [48]. The physical motivation of this problem is far away different of the corrugated waveguide, however and as we will see, the two different model belong to the same class of universality. The origin of the FAM back to earlier 1949 when Enrico Fermi [49] proposed a model in order to study the cosmic ray acceleration. After that, his original model was modified and studied using many different approaches [50-54]. A version which we address now, as it is shown in Figure 11, consists in a classical particle which is confined between two rigid walls and suffers elastic collisions with them. One of the walls is fixed at $x=l$ and the other one is periodically time varying $x_{w}(t)=\epsilon^{\prime} \cos (w t)$. The dynamics is described in terms of a two-dimensional nonlinear mapping for the variables $v_{n}$ and $t_{n}$, where the index $n$ corresponds to the $n$th collision with the moving wall. Before obtain the equations of the mapping, let us argue on a simplification commonly used. We will 


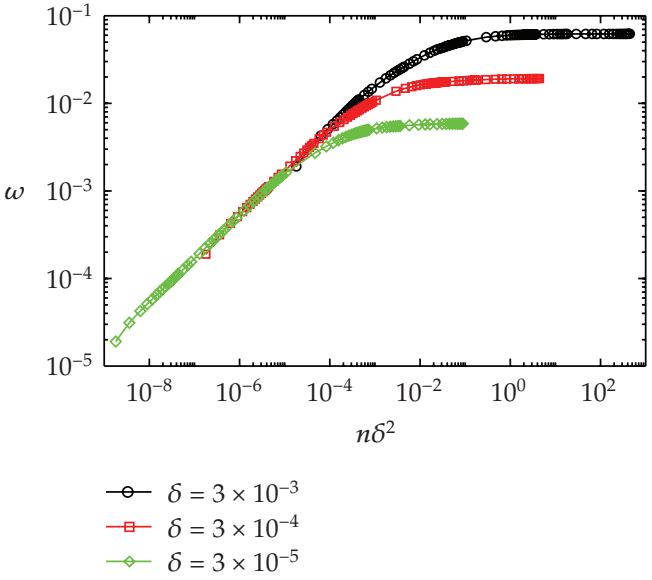

(a)



(b)

Figure 10: (a) Different curves of $\omega$ for three control parameters and (b) their collapse onto a single and universal plot.

suppose that both walls are fixed but that, when the particle suffers a collision with one of the walls, it exchanges momentum as if the wall was moving. This simplification carries the huge advantage of allowing us substantially to speed up the numerical simulations compared with the complete model (In the complete model, the movement of the oscillating wall is taken into account. Therefore, the instant of the collisions is obtained via a solution of a transcendental equation.) It is usefully applicable because the main dynamical properties of the system are preserved under such conditions. Incorporating this simplification in the model and using dimensionless variables $V_{n}=v_{n} /(w l), \epsilon=\epsilon^{\prime} / l$ and measuring the time in term of $\phi_{n}=w t_{n}$, the mapping is written as

$$
T:\left\{\begin{array}{l}
V_{n+1}=\left|V_{n}-2 \epsilon \sin \left(\phi_{n+1}\right)\right|, \\
\phi_{n+1}=\phi_{n}+\frac{2}{V_{n}} \quad \bmod 2 \pi .
\end{array}\right.
$$

The term $2 / V_{n}$ specifies the length of time during which the particle travels between collisions, while $-2 \epsilon \sin \left(\phi_{n+1}\right)$ gives the corresponding fraction of velocity gained or lost in the collision. The modulus function is introduced to avoid the particle leaving the region between the walls. We stress that the approximation of using the simplified FAM is valid in the limit of small $\epsilon$. In such a limit, the same transition observed for the periodically corrugated waveguide is observed in the FAM [48].

The phase space generated for iteration of the mapping (4.1) is shown in Figure 12. It is easy to see that there is a large chaotic sea surrounding KAM islands. The chaotic sea is also limited by an invariant spanning curve. This model exhibits also scaling properties near the phase transition from integrable to nonintegrable like those of the periodically corrugate waveguide. However, the observable $\omega$ is now obtained as

$$
\omega(n, \epsilon) \equiv \frac{1}{M} \sum_{j=1}^{M}\left[\sqrt{\bar{V}^{2}}(n, \epsilon)-\bar{V}_{j}^{2}(n, \epsilon)\right]
$$




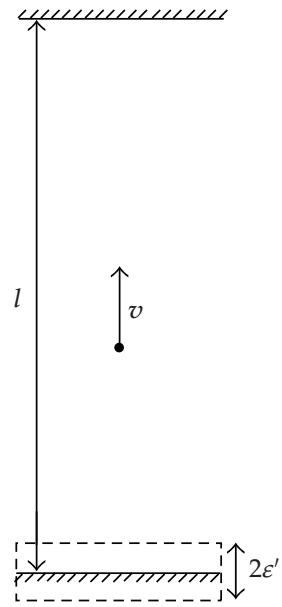

Figure 11: Illustrative picture of the one-dimensional Fermi accelerator model.

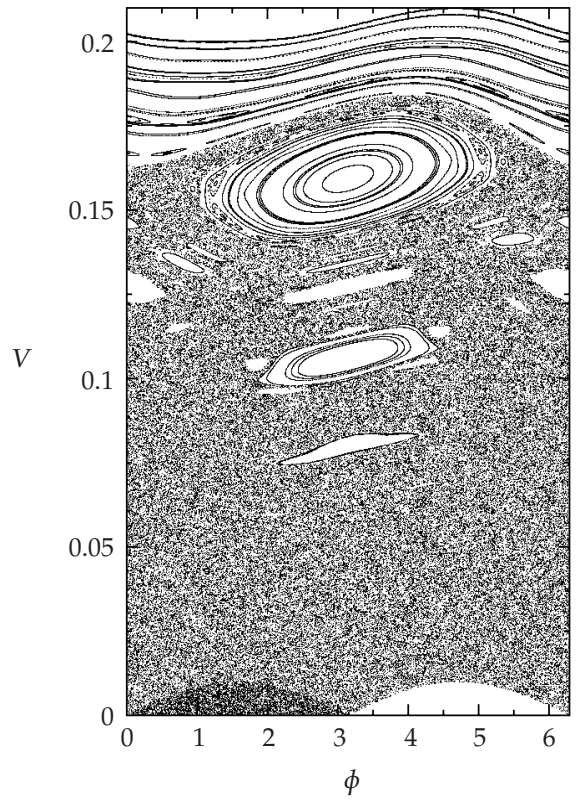

Figure 12: Phase space for the mapping (4.1) for the control parameter $\epsilon=10^{-2}$.

where the average velocity is defined as

$$
\bar{V}(n, \epsilon)=\frac{1}{n} \sum_{i=1}^{n} V_{i}
$$

and $M$ is an ensemble of different initial conditions.

It is shown in Figure 13(a), the behavior of $\omega$ for two different control parameters, while Figure 13(b) shows the curves of $\omega$ after a suitable transformation $n \rightarrow n \epsilon^{2}$. 


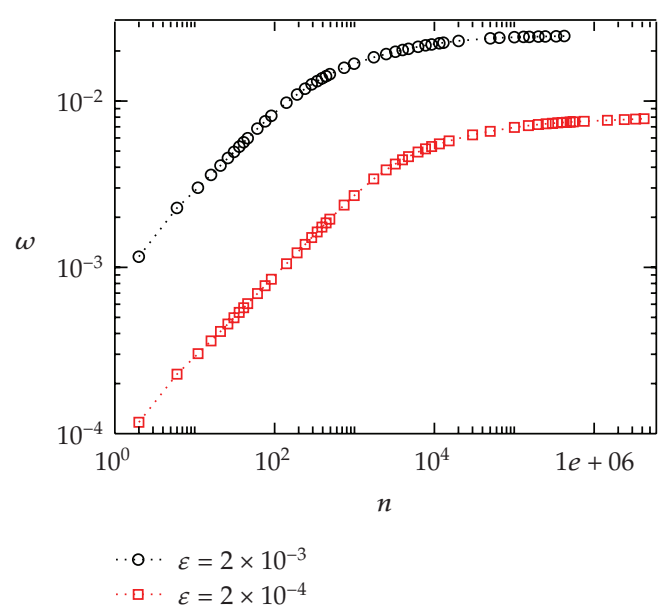

(a)

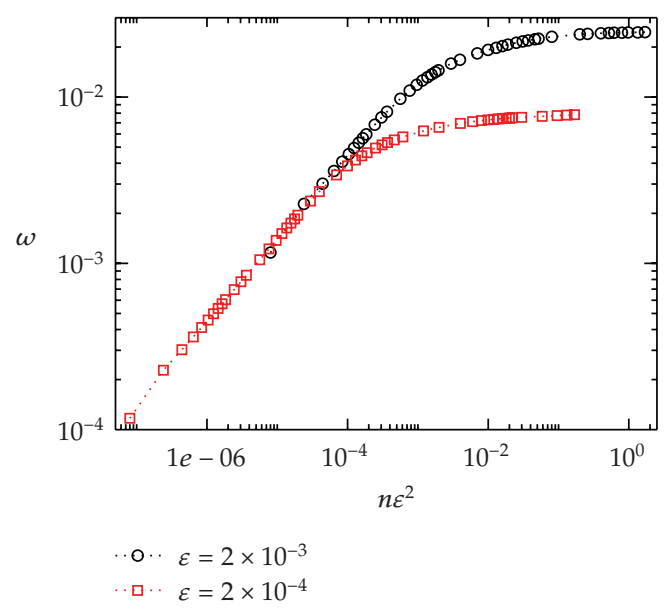

(b)

Figure 13: (a) Behavior of $\omega$ as a function of the iteration number $n$. (b) Behavior of $\omega$ as a function of $n \epsilon^{2}$. Both curves were derived from an ensemble average of $5 \times 10^{4}$ different initial conditions starting with $V_{0} \approx 0$.

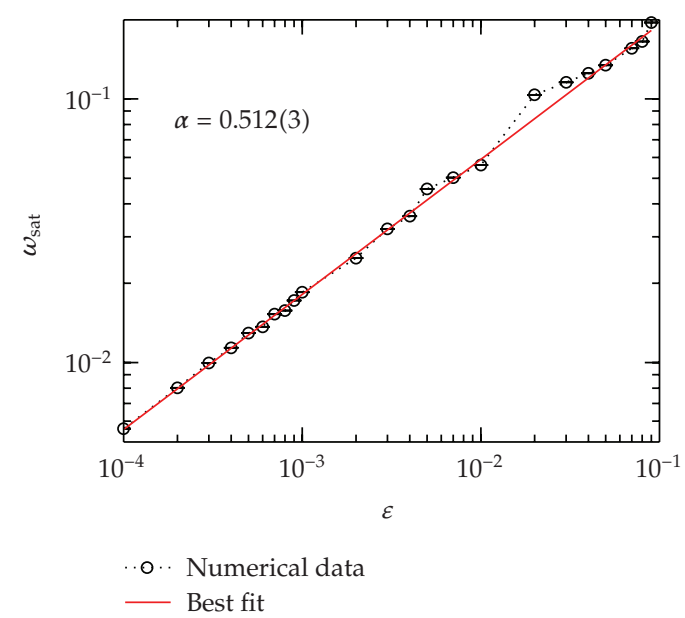

(a)

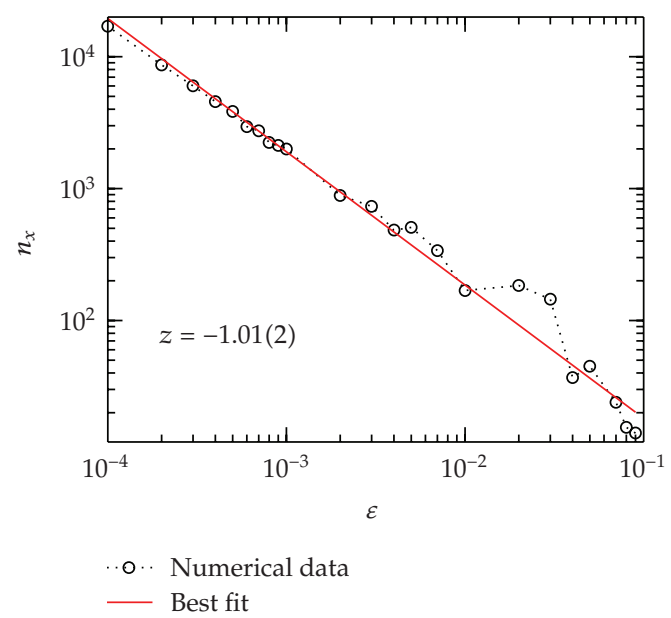

(b)

Figure 14: (a) Plot of $\omega_{\text {sat }}$ against the control parameter $\epsilon$. (b) The crossover iteration number $n_{x}$ as a function of $\epsilon$.

The exponent $\alpha$ is obtained in the asymptotic limit of large iteration number and it is independent of $V_{0}$. Figure 14(a) illustrates an attempt to characterize this exponent using the extrapolated saturation of $\omega$. Extrapolation is also required because, even after $10^{3} n_{x}$ iterations, $\omega$ has still not quite reached saturation. From a power law fit, we obtain $\alpha=0.512(3) \approx 1 / 2$. After averaging over different values of the control parameter $\epsilon$ in the range $\epsilon \in\left[10^{-4}, 10^{-1}\right]$, we then obtain $\beta=0.496(6) \approx 1 / 2$. It is found that the scaling relation for the exponent $z$ is $z=\alpha / \beta-2$. Considering the previous values of both $\alpha$ and $\beta$, it is found $z=-1$. The exponent $z$ can be also obtained numerically. Figure 14(b) shows the behavior of the crossover iteration number $n_{x}$ as function of the control parameter $\epsilon$. The power law fit gives us that $z=-1.01(2)$, in good accord with the scaling result. The scaling for $V_{0} \approx 0$ 


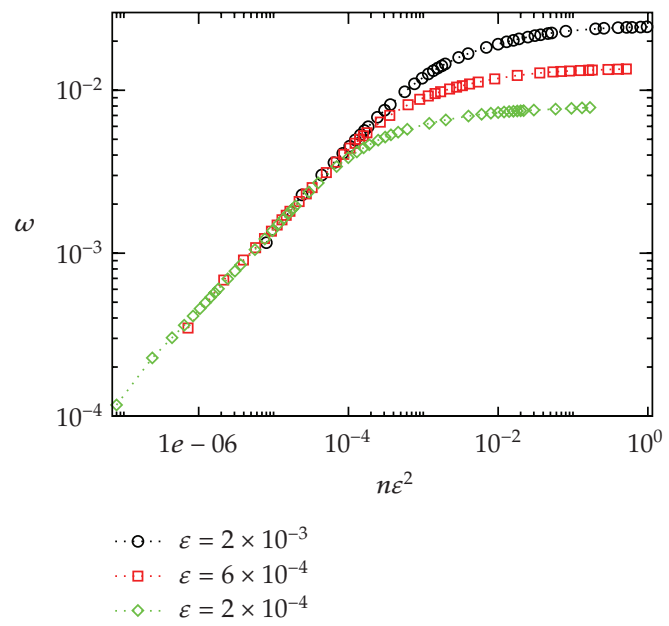

(a)

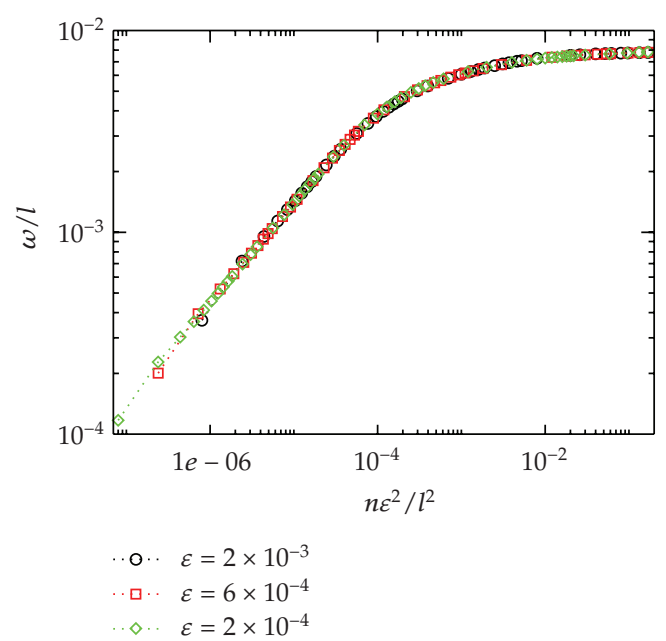

(b)

Figure 15: (a) Evolution of $\omega$ for different values of the control parameter $\epsilon$. (b) Collapse of the curves from (a) onto a universal curve. Both (a) and (b) were obtained using $V_{0} \approx 0$.

is demonstrated in Figure 15, where the three different curves for $\omega$ in Figure 15(a) are very well collapsed onto the universal curve seen in Figure 15(b).

Since the critical exponents are the same of those obtained for the corrugated waveguide, we can conclude that the one-dimensional Fermi accelerator model belongs to the same class of universality of the corrugated waveguide and experiences the same transition from integrability to nonintegrability when the control parameter $\epsilon$ goes from $\epsilon=0$ to $\epsilon \neq 0$. However the critical exponents obtained for a variant of a standard nontwist map are different from those obtained in Sections 3 and 4. Despite the similarities of the model presented in Section 2 and those models discussed in Sections 3 and 4, it belongs to a different class of universality from the corrugate waveguide and Fermi accelerator model since the critical exponents are rather different.

\section{Conclusion}

In summary, we have studied a phase transition from integrability to nonintegrability in three different two-dimensional mappings. Critical exponents for the behavior of the chaotic time series were obtained and used to define classes of universality. Even though the mappings are similar in their forms, the models discussed in Sections 3 and 4 belong to the same class of universality but then, they do not belong to the same class of universality of a variant of a standard nontwist map since the critical exponents are rather different. Indeed the term that seem to define the universality class is the expression of $K\left(I_{n+1}\right)$ (see (2.2)) and the exponent of the variable $I_{n+1}$ in that equation.

\section{Acknowledgments}

Financial support from CNPq, FAPESP and FUNDUNESP, Brazilian agencies are gratefully acknowledged. The present paper contains totally new results, those shown in Section 2 and also bring a short review of the articles [24], [25], and [48]. 


\section{References}

[1] G. A. Luna-Acosta, J. A. Méndez-Bermúdez, P. Seba, and K. N. Pichugin, "Classical versus quantum structure of the scattering probability matrix: chaotic waveguides," Physical Review E, vol. 65, no. 4, Article ID 046605, 8 pages, 2002.

[2] G. M. Zaslavsky, "Chaos, fractional kinetics, and anomalous transport," Physics Reports A, vol. 371, no. 6, pp. 461-580, 2002.

[3] D. G. Ladeira and J. K. L. da Silva, "Scaling properties of a simplified bouncer model and of Chirikov's standard map," Journal of Physics A, vol. 40, no. 38, pp. 11467-11483, 2007.

[4] R. Venegeroles, "Calculation of superdiffusion for the Chirikov-Taylor model," Physical Review Letters, vol. 101, no. 5, Article ID 054102, 4 pages, 2008.

[5] S. M. Soskin, R. Mannella, and O. M. Yevtushenko, "Matching of separatrix map and resonant dynamics, with application to global chaos onset between separatrices," Physical Review E, vol. 77, no. 3, Article ID 036221, 29 pages, 2008.

[6] F. R. N. Koch, F. Lenz, C. Petri, F. K. Diakonos, and P. Schmelcher, "Dynamical trapping and chaotic scattering of the harmonically driven barrier," Physical Review E, vol. 78, no. 5, Article ID 056204, 13 pages, 2008.

[7] J. E. Howard, A. J. Lichtenberg, M. A. Lieberman, and R. H. Cohen, "Four-dimensional mapping model for two-frequency electron cyclotron resonance heating," Physica D, vol. 20, no. 2-3, pp. 259$284,1986$.

[8] F. Saif, "Dynamical localization and signatures of classical phase space," Physics Letters A, vol. 274, no. 3-4, pp. 98-103, 2000.

[9] F. Saif, I. Bialynicki-Birula, M. Fortunato, and W. P. Schleich, "Fermi accelerator in atom optics," Physical Review A, vol. 58, no. 6, pp. 4779-4783, 1998.

[10] G. A. Luna-Acosta, K. Na, L. E. Reichl, and A. Krokhin, "Band structure and quantum Poincaré sections of a classically chaotic quantum rippled channel," Physical Review E, vol. 53, no. 4, pp. 32713283, 1996.

[11] A. M. O. de Almeida, Sistemas Hamiltonianos_Caos e Quantização, Editora da Unicamp, Unicamp, Brazil, 3rd edition, 1995.

[12] G. A. Luna-Acosta, G. Orellana-Rivadeneyra, A. Mendoza-Galván, and C. Jung, "Chaotic classical scattering and dynamics in oscillating 1-D potential wells," Chaos, Solitons E Fractals, vol. 12, no. 2, pp. 349-363, 2001.

[13] J. L. Mateos, "Traversal-time distribution for a classical time-modulated barrier," Physics Letters A, vol. 256, no. 2-3, pp. 113-121, 1999.

[14] E. D. Leonel and P. V. E. McClintock, “Chaotic properties of a time-modulated barrier," Physical Review E, vol. 70, no. 1, Article ID 016214, 11 pages, 2004.

[15] E. D. Leonel and P. V. E. McClintock, “Dynamical properties of a particle in a time-dependent doublewell potential," Journal of Physics A, vol. 37, no. 38, pp. 8949-8968, 2004.

[16] E. D. Leonel and P. V. McClintock, "Scaling properties for a classical particle in a time-dependent potential well," Chaos, vol. 15, no. 3, Article ID 033701, 7 pages, 2005.

[17] K. Ullmann and I. L. Caldas, "A symplectic mapping for the ergodic magnetic limiter and its dynamical analysis," Chaos, Solitons E Fractals, vol. 11, no. 13, pp. 2129-2140, 2000.

[18] I. L. Caldas, J. M. Pereira, K. Ullmann, and R. L. Viana, "Magnetic field line mappings for a tokamak with ergodic limiters," Chaos, Solitons E Fractals, vol. 7, no. 7, pp. 991-1010, 1996.

[19] S. S. Abdullaev and G. M. Zaslavsky, "Application of the separatrix map to study perturbed magnetic field lines near the separatrix," Physics of Plasmas, vol. 3, no. 2, pp. 516-528, 1996.

[20] A. Punjabi, H. Ali, and A. Boozer, "Symmetric simple map for a single-null divertor tokamak," Physics of Plasmas, vol. 4, no. 2, pp. 337-346, 1997.

[21] J. Koiller, R. Markarian, S. O. Kamphorst, and S. P. de Carvalho, "Static and time-dependent perturbations of the classical elliptical billiard," Journal of Statistical Physics, vol. 83, no. 1-2, pp. 127$143,1996$.

[22] A. Loskutov and A. Ryabov, "Particle dynamics in time-dependent stadium-like billiards," Journal of Statistical Physics, vol. 108, no. 5-6, pp. 995-1014, 2002.

[23] D. G. Ladeira and J. K. L. da Silva, "Scaling features of a breathing circular billiard," Journal of Physics A, vol. 41, no. 36, Article ID 365101, 13 pages, 2008.

[24] E. D. Leonel, “Corrugated waveguide under scaling investigation," Physical Review Letters, vol. 98 , no. 11, Article ID 114102, 4 pages, 2007. 
[25] A. F. Rabelo and E. D. Leonel, "Finding invariant tori in the problem of a periodically corrugated waveguide," Brazilian Journal of Physics, vol. 38, no. 1, pp. 54-57, 2008.

[26] A. L. Virovlyansky and G. M. Zaslavsky, "Evaluation of the smoothed interference pattern under conditions of ray chaos," Chaos, vol. 10, no. 1, pp. 211-223, 2000.

[27] I. P. Smirnov, A. L. Virovlyansky, and G. M. Zaslavsky, "Theory and applications of ray chaos to underwater acoustics," Physical Review E, vol. 64, no. 3, Article ID 036221, 20 pages, 2001.

[28] A. Iomin and Yu. Bliokh, "Wave localization as a manifestation of ray chaos in underwater acoustics," Communications in Nonlinear Science and Numerical Simulation, vol. 8, no. 3-4, pp. 389-399, 2003.

[29] B. Huckestein, R. Ketzmerick, and C. H. Lewenkopf, "Quantum transport through ballistic cavities: soft vs hard quantum chaos," Physical Review Letters, vol. 84, no. 24, pp. 5504-5507, 2000.

[30] B. Huckestein, R. Ketzmerick, and C. H. Lewenkopf, "Erratum: quantum transport through ballistic cavities: soft vs hard quantum chaos," Physical Review Letters, vol. 87, no. 11, Article ID 119901, 2 pages, 2001.

[31] L. P. Kouwenhoven, F. W. J. Hekking, B. J. van Wees, C. J. P. M. Harmans, C. E. Timmering, and C. T. Foxon, "Transport through a finite one-dimensional crystal," Physical Review Letters, vol. 65, no. 3, pp. 361-364, 1990.

[32] M. Leng and C. S. Lent, "Recovery of quantized ballistic conductance in a periodically modulated channel," Physical Review Letters, vol. 71, no. 1, pp. 137-140, 1993.

[33] G. A. Luna-Acosta, J. A. Méndez-Bermúdez, and F. M. Izrailev, "Periodic chaotic billiards: quantumclassical correspondence in energy space," Physical Review E, vol. 64, no. 3, Article ID 036206, 18 pages, 2001.

[34] G. A. Luna-Acosta, J. A. Méndez-Bermúdez, and F. M. Izrailev, “Quantum-classical correspondence for local density of states and eigenfunctions of a chaotic periodic billiard," Physics Letters A, vol. 274, no. 5-6, pp. 192-199, 2000.

[35] G. B. Akguc and L. E. Reichl, "Direct scattering processes and signatures of chaos in quantum waveguides," Physical Review E, vol. 67, no. 4, Article ID 046202, 10 pages, 2003.

[36] R. B. Hwang, "Negative group velocity and anomalous transmission in a one-dimensionally periodic waveguide," IEEE Transactions on Antennas and Propagation, vol. 54, no. 2, pp. 755-759, 2006.

[37] L. A. Bunimovich, "On the ergodic properties of nowhere dispersing billiards," Communications in Mathematical Physics, vol. 65, no. 3, pp. 295-312, 1979.

[38] Y. G. Sinai, “Dynamical systems with elastic reflections," Russian Mathematical Surveys, vol. 25, no. 2, pp. 137-189, 1970.

[39] M. V. Berry, "Regularity and chaos in classical mechanics, illustrated by three deformations of a circular "billiard"," European Journal of Physics, vol. 2, no. 2, pp. 91-102, 1981.

[40] S. O. Kamphorst and S. P. de Carvalho, "Bounded gain of energy on the breathing circle billiard," Nonlinearity, vol. 12, no. 5, pp. 1363-1371, 1999.

[41] M. Robnik, "Classical dynamics of a family of billiards with analytic boundaries," Journal of Physics A, vol. 16, no. 17, pp. 3971-3986, 1983.

[42] M. Robnik and M. V. Berry, "Classical billiards in magnetic fields,” Journal of Physics A, vol. 18, no. 9, pp. 1361-1378, 1985.

[43] R. Markarian, S. O. Kamphorst, and S. P. de Carvalho, "Chaotic properties of the elliptical stadium," Communications in Mathematical Physics, vol. 174, no. 3, pp. 661-679, 1996.

[44] J.-P. Eckmann and D. Ruelle, "Ergodic theory of chaos and strange attractors," Reviews of Modern Physics, vol. 57, no. 3, pp. 617-656, 1985.

[45] A. J. Lichtenberg and M. A. Lieberman, Regular and Chaotic Dynamics, vol. 38 of Applied Mathematical Sciences, Springer, New York, NY, USA, 2nd edition, 1992.

[46] E. D. Leonel and P. V. E. McClintock, "A hybrid Fermi-Ulam-bouncer model," Journal of Physics A, vol. 38, no. 4, pp. 823-839, 2005.

[47] A.-L. Barabási and H. E. Stanley, Fractal Concepts in Surface Growth, Cambridge University Press, Cambridge, UK, 1985.

[48] E. D. Leonel, P. V. E. McClintock, and J. K. L. da Silva, “Fermi-Ulam accelerator model under scaling analysis," Physical Review Letters, vol. 93, no. 1, Article ID 014101, 4 pages, 2004.

[49] E. Fermi, "On the origin of the cosmic radiation," Physical Review, vol. 75, no. 8, pp. 1169-1174, 1949.

[50] M. A. Lieberman and A. J. Lichtenberg, "Stochastic and adiabatic behavior of particles accelerated by periodic forces," Physical Review A, vol. 5, no. 4, pp. 1852-1866, 1972. 
[51] L. D. Pustyl'nikov, “On Ulam's problem," Theoretical and Mathematical Physics, vol. 57, no. 1, pp. 10351038, 1983.

[52] A. J. Lichtenberg, M. A. Lieberman, and R. H. Cohen, “Fermi acceleration revisited," Physica D, vol. 1, no. 3, pp. 291-305, 1980.

[53] G. Karner, "The simplified Fermi accelerator in classical and quantum mechanics," Journal of Statistical Physics, vol. 77, no. 3-4, pp. 867-879, 1994.

[54] S. T. Dembiński, A. J. Makowski, and P. Pepłowski, “Quantum bouncer with chaos,” Physical Review Letters, vol. 70, no. 8, pp. 1093-1096, 1993. 


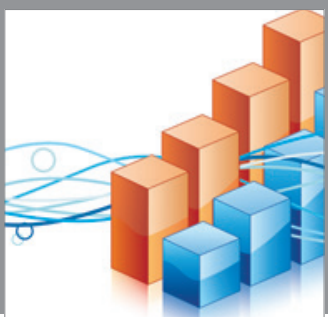

Advances in

Operations Research

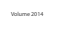

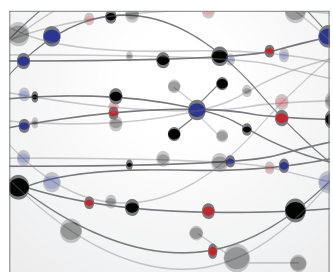

\section{The Scientific} World Journal
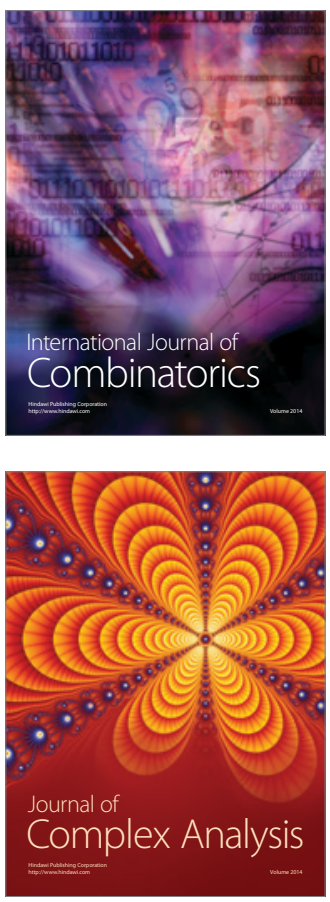

International Journal of

Mathematics and

Mathematical

Sciences
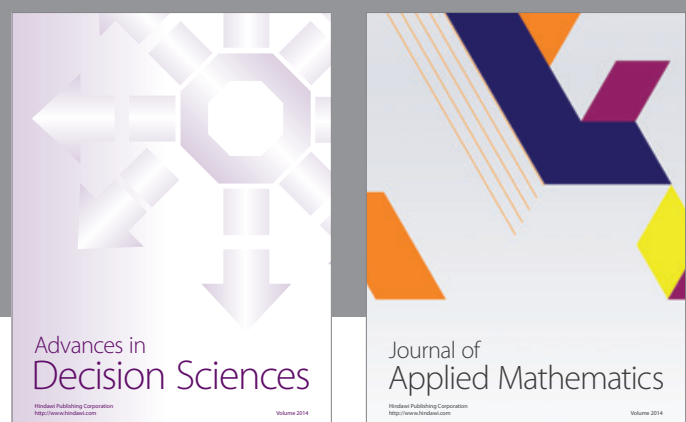

Journal of

Applied Mathematics
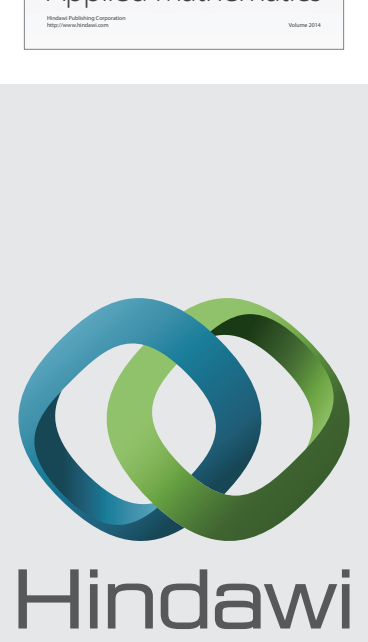

Submit your manuscripts at http://www.hindawi.com
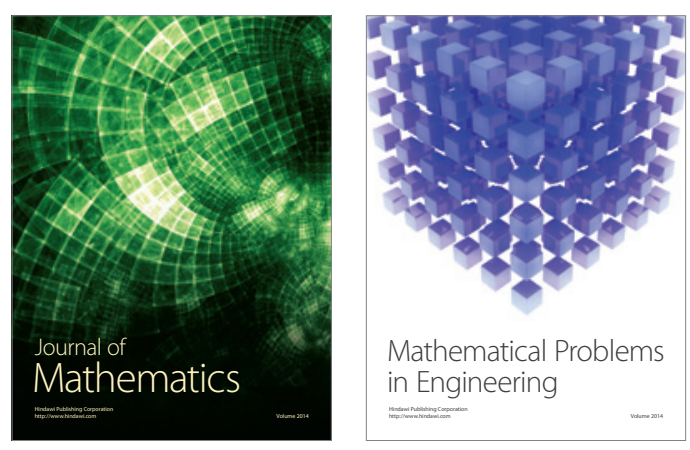

Mathematical Problems in Engineering
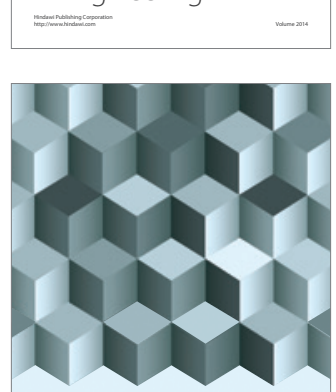

Journal of

Function Spaces




ournal of

Probability and Statistics

Promensencen
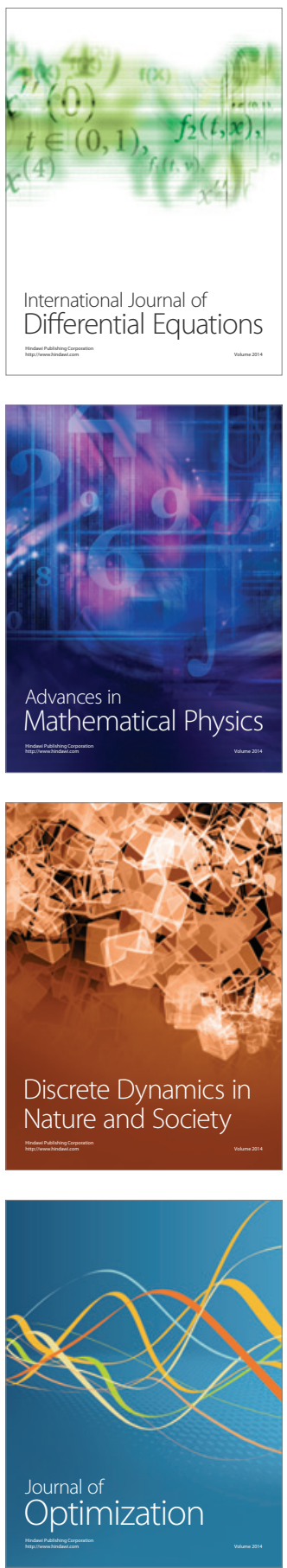